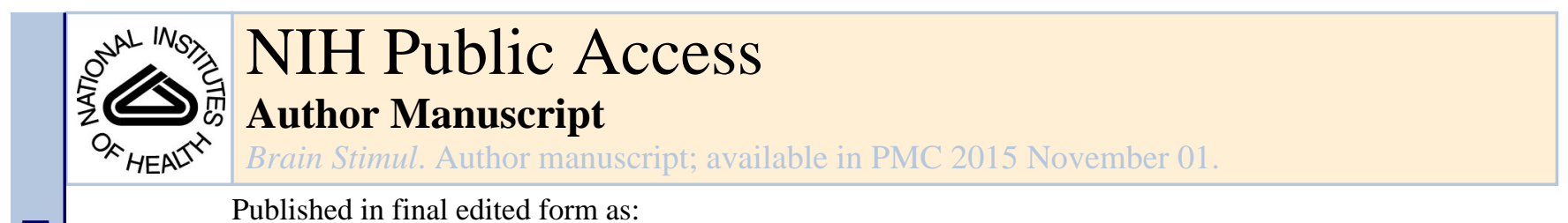

Published in final edited form as:

Brain Stimul. 2014 ; 7(6): 784-792. doi:10.1016/j.brs.2014.07.035.

\title{
The effects of anodal stimulation of the left prefrontal cortex on sentence production
}

\author{
Nazbanou Nozari ${ }^{1}$, Jennifer E. Arnold ${ }^{2}$, and Sharon L. Thompson-Schill ${ }^{3}$ \\ ${ }^{1}$ Department of Neurology, Johns Hopkins University School of medicine \\ ${ }^{2}$ Department of Psychology, University of North Carolina at Chapel-Hill \\ ${ }^{3}$ Department of Psychology, University of Pennsylvania
}

\begin{abstract}
Background-Most studies in which Anodal Transcranial Direct Current Stimulation (A-tDCS) has been used to improve language production have focused on single words. Yet sentence production requires more than lexical retrieval. For example, successful suppression of the past and careful planning of the future are two critical requirements for producing a correct sentence. Can A-tDCS improves those, and by extension, production at the sentence level?
\end{abstract}

Objective/Hypothesis-Given that many aspects of sentence production beyond word retrieval require frontally-mediated operations, we hypothesized that A-tDCS to the left prefrontal cortex should benefit various operation involved in producing sentences, two of which, suppression of the past and planning of the future, were targeted in this study.

Methods-We used a paradigm that elicited construction of sentences through event description, but was structured enough to allow for between-subject comparison, clear error identification, and implementation of experimental manipulations to probe certain aspects of production.

Results-We showed that A-tDCS to the left PFC reliably decreased the number of incomplete and errorful sentences. When the origin of this improvement was probed, we found that A-tDCS significantly decreased errors due to premature commitment to the future word (insufficient internal monitoring), and had a marginal effect on errors of perseverations (insufficient suppression of the past).

Conclusion-We conclude that A-tDCS is a promising tool for improving production at the sentence level, and that improvement can be expected when internal monitoring and control over verbal responses is impaired, or for certain cases of perseveratory errors.

(02014 Elsevier Inc. All rights reserved.

Corresponding Author: Nazbanou Nozari, nozari@jhu.edu, Address: Neurology Department, Johns Hopkins University School of Medicine, 1629 Thames Street, Suite 350, Baltimore, MD 21231, Phone: 443-287-1712, Fax: 410-955-0188.

Conflict of interest statement: None of the authors has a conflict of interest.

Publisher's Disclaimer: This is a PDF file of an unedited manuscript that has been accepted for publication. As a service to our customers we are providing this early version of the manuscript. The manuscript will undergo copyediting, typesetting, and review of the resulting proof before it is published in its final citable form. Please note that during the production process errors may be discovered which could affect the content, and all legal disclaimers that apply to the journal pertain. 


\section{Keywords}

sentence production; Prefrontal cortex; Transcranial direct current stimulation (tDCS); language; speech errors; perseveration

\section{Introduction}

Transcranial Magnetic Stimulation (TMS) and Transcranial Direct Current Stimulation (tDCS) are two methods of non-invasive brain stimulation currently used for modifying the processing of language, as well as other cognitive functions. Of the two, tDCS has gained much popularity in recent years, because it can be safely administered over a period of several minutes (1), and does not have some of the unpleasant side effects of TMS such as twitching of the facial muscle due to unwanted stimulation of the trigeminal nerve, when applied to frontal areas. Weak electrical current in tDCS modulates neural activity, by either depolarizing or hyperpolarizing neuronal membranes, depending on the polarity of the current $(2,3)$. Clinically, anodal stimulation is often used when the goal is to excite the neural tissue and to improve performance, while cathodal stimulation is employed to inhibit neuronal activation which, in most cases, is expected to deteriorate performance. While this assumption is not universally true, especially when stimulation effects are examined in a cognitive, as opposed to a simple motor, task (4-6), it is consistent with most of the reports. Especially, anodal tDCS is often reported to facilitate cognitive processes (e.g., 6,7).

In this paper, we focus on the effects of anodal tDCS on language production. Several studies over the past decade have shown promising results of anodal tDCS on word retrieval and new vocabulary learning, in both neurologically-intact and brain-damaged individuals (e.g., 8-12). However, there has been much less work on the effects of stimulation at the level of sentence production. We present the first systematic effort in studying the effects of anodal stimulation of the left prefrontal cortex (PFC) on the accuracy of production at the sentence level. Our purpose is two-fold: (a) to introduce a paradigm that allows for generation of sentences in an ecologically-valid way (i.e., describing events after they are semantically processed), yet with an imposed structure that would make between-participant comparisons possible, and to show the benefits of such an approach, and (b) to show that stimulation could have beneficial effects at the level of sentence production that go beyond isolated lexical retrieval, a finding that could expand its clinical applicability.

\section{Anodal tDCS and language}

There is now plenty of evidence that anodal stimulation of the left hemisphere has beneficial effects on language production (13-16). Fertonani et al. (2010) showed that anodal stimulation of the left dorsolateral PFC improves picture naming performance by decreasing response latencies. A similar effect was found using anodal stimulation of the left posterior peri-sylvian region, which includes Wernicke's area (17). Not surprisingly, improvement in the accuracy of lexical retrieval has not been reported when neurotypical participants named pictures, because such individuals rarely make errors in this task. An exception was the study of proper noun retrieval, in which participants were prompted to name 65 pictures of famous people (18). Authors found that for trials with longer response latencies, anodal 
stimulation of the right anterior temporal lobe increased naming accuracy by $11 \%$.

Spontaneous word generation also benefits from anodal stimulation. Verbal fluency is an example (1). Iyer and colleagues (1) showed improvement of phonological verbal fluency (generating more words that started with a target letter), when left PFC was stimulated with $2 \mathrm{~mA}$ anodal tDCS. Anodal tDCS can also help with language learning/re-learning. Improved associative learning has been demonstrated for both nouns and verbs $(4,8,10,11,19-22)$, and one study has reported improved learning of artificial grammar (23).

Recently, examining the effects of anodal tDCS has been extended from single word production to production of multi-word utterances, as in tongue-twisters $(24,25)$, and one recent study has explored the beneficial effects of anodal stimulation on conversational speech (26). Marangolo and colleagues (26) paired 20 minutes of $1 \mathrm{~mA} \mathrm{A-tDCS} \mathrm{over} \mathrm{the}$ Broca's area with intensive conversation therapy on 12 individuals with nonfluent aphasia. Therapy consisted of 10 sessions, during which the patient and the therapist viewed three 15-minute video clips of everyday events, and the therapist helped the patient describe the events using a variety of cues (gestures, drawings, orthographic or phonological cues).

Another set of three 15-minute clips was used for testing, in which the patient was prompted to describe the events without cues from the therapist. Anodal stimulation of Broca's -- but not Wernicke's -- area significantly increased patients' use of verbs, informative fragments without verbs, and sentences.

Marangolo et al.'s (26) results provide a serious motivation for more structured studies of tDCS effects on sentence production. Quite possibly the usefulness of tDCS is not limited to lexical retrieval or associative learning of isolated items, and instead tDCS may lead to additional benefits in other areas of sentence production. Sentence production differs from single-word retrieval in a number of ways. Here, we focus on two operations necessary for correct production of a sentence, namely, successful suppression of the past, and careful planning of the future (27). Both of these are operations carried out by the executive system. Thus, it is plausible to hypothesize that PFC stimulation could benefit sentence production beyond lexical retrieval, by improving these two crucial functions, among possible others.

\section{A more systematic approach to studying stimulation effects in sentence production}

While Marangolo et al.'s (26) results provide support for the idea that left PFC stimulation could benefit sentence production, that study used unstructured conversational speech. We propose that complementing that approach with a more structured approach has several benefits: (a) It affords the possibility of between-individuals comparison at any given point in time: While studying free conversations has undeniable value for measuring progress over time (because each patient's baseline performance is his/her own standard of comparison), unstructured conversations are not ideal for cross-sectional comparisons between individuals, because different speakers may produce very different amount of speech, with very different structures. (b) It allows for the possibility of studying the change in the probability of errors. Earlier, we pointed out the difficulty in assessing the effects of tDCS on the accuracy of lexical retrieval in healthy adults, because they rarely make errors on the names of familiar objects when they are presented in isolation. However, speech errors are not uncommon in sentence production. (c) Importantly, a paradigm that forces some 
systematicity into the sentence production process not only elicits errors, but provides identifiable targets for those errors (i.e., what the participant should have said instead of the error), and ways to elicit specific types of errors. This, as we show below, enables us to test specific hypotheses regarding the effects of stimulation.

In the paradigm used in this study, participants had to produce sentences that described events involving one (or two) of eight geometric shapes performing one of five simple actions. Because the number of possible events is very large, producing sentences must be on the fly and through independent processing of each event, as is the generation of sentences in most everyday life events. However, the generated sentences pertaining to each visual event are highly similar across individuals. We discussed earlier that production of a sentence requires successful suppression of past words, and good planning of the future words. A failure of the former results in errors of perseveration, a problem common in some patients with aphasia. We traced errors made on trial $t$, back to trial $t-l$ to examine if the error is due to perseverance of a recently-uttered word. We also probed a specific aspect of future planning, that of premature commitments. A premature commitment happens when internal monitoring processes fail to inhibit production before enough information is available. To probe this, we designed two of the five possible motions (jumping over an object, and looping around an object) to be visually identical up to a certain point (a halfcircle). When faced with the start of a circular motion, the optimal planning strategy would thus be to hold production until the point of disambiguation.

Twenty-four participants were randomly assigned to anodal and sham stimulation of left PFC and each viewed 134 visual events. We chose the left PFC because several studies have shown improved verbal production through anodal stimulation of this area (e.g., $9,12,19,20,23,26)$. Moreover, the left PFC activation has been shown in neuroimaging studies when participants produce sentences (28), but not single unrelated words (29), and one study using Voxel-based Lesion Symptom Mapping demonstrated the specific contribution of this region to sentence production when lexical retrieval abilities were factored out (30). We compared performance in A-tDCS and sham conditions on the number of incomplete and errorful sentences. We then probed the origin of difference in error probabilities by comparing perseverations and premature commitments between the two groups.

\section{Methods}

\section{Participants}

Twenty-four ${ }^{1}$ (13 females) right-handed, native speakers of English participated in the experiment and received $\$ 20$ for their participation. Participants in the anodal and sham groups were matched on age and education (age: mean $=21.8, \mathrm{SD}=2.3$ in anodal vs. mean $=21.2, \mathrm{SD}=3.1$ in sham; $\mathrm{t}(22)=1.2, \mathrm{p}=0.24$; education: mean $=15.0, \mathrm{SD}=1.4$ in anodal vs. mean $=14.3, \mathrm{SD}=1.3$ in sham; $\mathrm{t}(22)=.60, \mathrm{p}=0.55)$. All participants gave written

\footnotetext{
${ }^{1}$ One subject was replaced because she could not keep up with the pace of visual actions, and this was reflected in her Speed Test, described in Appendix B.
} 
consent for tDCS administration in accordance with the IRB-approved experimental protocol.

\section{Materials}

One hundred and thirty four visual events were presented using Microsoft Powerpoint. Figure 1 shows an example of the screen as participants viewed it. The screen consisted of a black grid on a white background, with eight geometrical objects. Two versions were tested with different objects and colors. Objects and colors in version 1 are depicted in Figure 1. Version 2 (not pictured) consisted of two squares, two triangles, two pentagons and two ovals; the colors used were pink, yellow, gray and blue. Object and color names across the two versions were matched in frequency and number of syllables. The rationale for using two sets of materials was to introduce more diversity into spoken materials and make conclusions less item-dependent. There were five possible actions in the experiment. Appendix A provides a list of the five motions with examples, as well as a complete list of the 134 trials in Version 1. While the two versions differed in shapes and color, they were otherwise identical (i.e., the relative position of objects and their actions were the same). On each trial only one object performed a single action at the pace of $21 \mathrm{~mm} / \mathrm{sec}$. (This speed was determined based on a pilot study, the details of which are described in Appendix B.) Actions were independent of one another and the sequence was random (i.e., one action did not cue the next). The 134 trials were divided into 27 sets, with breaks in between sets. Each set contained 4-6 trials.

\section{Procedure}

Participants were randomly assigned to anodal and sham conditions and administration of Versions 1 and 2 was counterbalanced across conditions. Participants sat comfortably at a distance of 25 inches from a 19-inch monitor. They first completed a 2-minute speed test (see Appendix B). Then, stimulation was initiated. During orientation, participants first viewed - and named - each of the eight experimental objects with its color. Next, the five motions were taught individually and participants practiced examples for each. They then viewed a set of four sentences, similar to the experimental design, and listened to a recording describing each motion as it happened. Next they reviewed the same example (without the description) and provided their own descriptions. Problems, if any, were corrected and they completed two more event sets as practice. They were instructed to describe the events as they were taking place (like a sports reporter) and were discouraged from recalling sentences from memory (See also Appendix B for details of the speed test, the orientation phase, and the practice phase). The main experiment started after two minutes of break. Participants initiated each of the 27 sets by pressing the space bar, but could not pause within a set. This means that they had to speak 4-6 consecutive sentences before a break. All participants completed the entire experiment (on average 5-6 minutes of orientation and practice, 2 minutes of break, and 11-12 minutes of experimental trials without feedback) during stimulation.

\section{Direct Current Stimulation}

Saline-soaked sponge electrodes with a surface area of $25 \mathrm{~cm}^{2}$ were used to deliver direct current generated by a continuous current stimulator (Magstim Eldith 1 Channel DC 
Stimulator Plus, Magstim Company Ltd., Whitland, Wales). During stimulation, $1.5 \mathrm{~mA}$ direct current (with a 30-second ramp up and ramp down) was applied for 20 minutes. Stimulation was applied for only 30 seconds during sham. The anode was placed over F3, according to the 10-20 international system for EEG electrode placement. The cathode was placed over the right supraorbital region (7). According to a post-tDCS questionnaire, all participants reported that they believed they had received stimulation. Stimulation was started at the beginning of the orientation phase and continued for 5-6 minutes on average (with a 2 minute break to follow) before participants started the experimental trials. The reason for this choice was (a) to give anodal tDCS a chance to affect learning, as it has been suggested to do so through modulation of NMDA receptors (31), and (b) to allow enough time for stimulation effects to cause visible effects on behavior.

\section{Statistical Analysis}

All the models used in the current paper are multilevel logit models with random effects of subjects and items (e.g., 32, 33). These models are preferable for all analyses where results are to be generalized from sample subjects to the population, and from the task items to other sentences, as the random structure of the model prevents the variance that is truly due to random subjects and items to be attributed to the variable of interest (See appendix C for an extended discussion of superiority of mixed models over ANOVA).

Because we are interested in sentence accuracy, our dependent variable of interest is accuracy at the sentence -- and not the word -- level. This was coded as a binary variable (a sentence is either errorful or error-free). The independent variable of interest is always stimulation, with two levels: anodal and sham. Following Barr and colleagues (34), the random effect structure has been kept maximal. This included the random intercepts for subjects (are some subjects more error-prone?), random intercept of items (are some sentences more error-prone?), and the random slope of stimulation over items (do some sentences benefit more from tDCS?). Note that because stimulation is manipulated between subjects, random slope of stimulation over subject is not included in the model. The models produce coefficients, $\mathrm{z}$ statistics, and corresponding $\mathrm{p}$-values, and the results can be interpreted as in regression models (Appendix D).

\section{Results}

Fails

A sentence was defined as a Fail if it did not have a verb, and was not an appropriate ellipsis. There were 38 Fails in the anodal and 65 Fails in the Sham condition. Figure 2 shows the average number of Fails per condition. This difference was significant $(\mathrm{z}=2.96, \mathrm{p}$ $=0.004)$. Table D1 in Appendix D presents the full results of this analysis. Fail trials were excluded from all subsequent analyses.

\section{Errors}

As explained above, we are interested in sentence-level accuracy. However we must first ensure that using this grain of analysis does not misrepresent the actual error probabilities. For example, if sham and anodal conditions have 100 and 70 errorful sentences respectively, 
but each errorful sentence contains only one error in sham, but two errors in anodal, using sentence-level analysis would be misleading, because the error probability would in fact be higher in anodal than sham. Table 1 shows the number of sentences with one, two, and three errors in each condition. As can be seen in the table, in all three categories there are more errorful sentences in sham. This means that using a sentence-level analysis is in fact conservative, and results would only be stronger if a word-level analysis was to be performed.

There were a total of 220 errorful sentences in the anodal and 379 errorful sentences in the sham condition (note that these numbers are lower than those in Table 1, because sentences with two and three errors are counted only once). Figure 3 (upper panel) shows the average proportion of errors in each condition. The results of the analysis are presented in Table D2 in Appendix D. The difference in the probability of errorful sentences was significant $(\mathrm{z}=$ $2.55, \mathrm{p}=0.01$ ). In fact, anodal stimulation reduced the number of errorful sentences by a factor of two $(\exp (0.72)=2.05)$. Figure 3 (lower panel) shows the average proportion of errorful sentences plotted separately for each word type. The purpose of the graph is to show that all word types benefited from stimulation, and the effect was not driven by a specific class of words (e.g., verbs).

So far, we have shown that anodal stimulation significantly decreases the number of errorful sentences. Next, we ask how this benefit is achieved. We probe two types of errors (perseverations and premature commitments) which we earlier argued point to two processes important for sentence production. There are four possible outcomes: (1) A-tDCS reduces perseverations from the past, (2) A-tDCS reduces premature commitments to the future. (3) A-tDCS affects both processes. (4) A-tDCS affects processes altogether different from the two evaluated here.

Premature commitments-A premature commitment was coded when "jumping over" was produced in place of "looping around" and vice versa. The two actions are indistinguishable up to a certain point ("jumping over" stops at a half-circle, but "looping around" continues). When the speaker commits to a verb before the point of disambiguation has been reached, it is likely to result in an error due to premature commitment. There were 44 such errors in the anodal condition and 109 in sham. In calculating error opportunities, we only included those trials that contained either a "jumping over" or a "looping around" target verb. Although there were more "jumping over" than "looping around" sentences in the design (42 vs. 32), this was not reflected in participants' production preferences. In fact, of the total 153 premature commitments, 69 were erroneous use of "jump" and 84 were erroneous use of "loop", implying a slightly higher preference for producing "loop". Therefore, frequency was not a driving factor in making these types of errors. For our purpose, the important point was the ambiguity of the situation at the onset, which clearly led to errors on both verbs.

Perseveration-An error was marked as perseveratory if it was an incorrect repetition of a word previously spoken in the same sentence or one sentence before ${ }^{2}$. There were 58 perseverations in the anodal and 119 perseverations in the sham condition. Opportunities for perseveration are all the sentences that a participant has uttered (Fails are removed). 
To avoid including the same trial in both error categories, we removed all trials in which a "jumping over"/"looping around" error could also be considered a perseveration from the previous trial. Figures $4 \mathrm{a}, \mathrm{b}$ shows the average proportion of sentences with premature commitments and perseverations after removing the ambiguous trials (raw error counts are presented in Table 2). Tables D3 and D4 in Appendix D present the results of the analyses. Anodal stimulation significantly reduced the number of premature commitments $(\mathrm{z}=3.14, \mathrm{p}$ $=0.002)$, and marginally decreased perseveratory errors $(\mathrm{z}=1.74, \mathrm{p}=0.08)$.

\section{General Discussion}

This investigation represents a first step towards the systematic study of the effects of anodal tDCS on sentence production. We showed that the effects of anodal tDCS over left PFC, previously shown to benefit word-level production and learning, can be extended to sentence production. We developed a paradigm that elicits sentence production from meaning, but that has enough structure to allow for between-subject comparison, accurate error identification, error-source tracking, and implementation of experimental manipulations to study specific aspects of production. Our results showed that anodal stimulation of the left PFC significantly decreased the number of incomplete (Fails) and errorful sentences, and sentences with errors. We then targeted two operations necessary for error-free sentence production: successful suppression of the past and controlled planning of the future.

Failure to suppress the past results in errors of perseveration, a well-known problem in certain aphasic patients (35). Our results suggest that PFC stimulation could help reduce this type of error, possibly by biasing competition towards the relevant, and away from the irrelevant, word (e.g., 25). This effect, however, was only marginal. The fact that tDCS did not reliably decrease perseveration errors might be of theoretical importance: recently, it has been suggested that more than one mechanism contributes to perseverations. In the domain of spelling, Fischer-Baum and Rapp (36) showed that in some of their patients who produced perseveratory errors, the problem was not a failure to inhibit the previous response, but instead a failure to properly activate the current response. Activation of the current word can happen independently of the PFC (29), through the process of spreading activation in the relevant language module (e.g., the temporal cortex for semantic-lexical mapping). Current results are compatible with the view that failure to inhibit the past response is not the only determining factor in perseverations, and improvement can only be expected to the degree that this specific problem is contributing to perseveration errors, but it might not help patients whose main deficit is activating the current response due to noisy and damaged production modules (e.g., 37).

Planning the future is more multi-faceted, and can be tested in several ways. The aspect of future planning that we investigated was the ability to withhold a response until the visual ambiguity has been resolved, which constitutes better internal monitoring of the language plan (e.g., (38)). Our results showed that anodal tDCS reliably increased this ability. This

\footnotetext{
${ }^{2}$ Note that this criterion is to some degree arbitrary, because we could have chosen two, three or $n$ sentences before. We chose one sentence back because given the limited number of objects, colors and motions used in this experiment choosing a range wider than 1back would force more errors into the perseveratory category, simply because it increases the chance of appearance of an item that the perseveration could potentially be attributed to, even if the occurrence of the error has been independent of this item.
} 
finding could have implications for treatment of deficits where internal monitoring is a major problem, such as the Gilles de La Tourette Syndrome (39). In summary, our results demonstrate that tDCS effects on sentence production can, and should be, studied in a more systematic way, as the effects seem to go well beyond isolated lexical production. This is particularly promising, because it points to the possibility of applying tDCS to conditions where the problem is not simply in lexical retrieval, but in other aspects of production, such as monitoring and control.

\section{Acknowledgments}

This work was supported by R01-DC009209. We would like to thank Elizabeth Schopfer, Kristina Woodard, William Marinello, Luke Miller, Michael Chen, Sarah Lineberry, and Ivy Hauser for their help with data collection, transcription and coding.

\section{Appendix A}

\section{Experimental materials}

The list of the five possible motions with examples:

1. Moving. One object moved on a straight line. The number of blocks (on the grid) and the direction of the movement were to be described. For example: "The red diamond moves two blocks down". The maximum travel distance for an object was 3 blocks (hence removing the need for explicitly counting blocks). Participants were informed about this. Moving always involved a single object.

2. Jumping over. One object jumped over another object (this motion created a semicircle). This action always covered the same distance, and direction did not have to be described. For example: "The brown circle jumps over the purple rectangle."

3. Looping around. One object made a full circle around another object. Similar to jumping over, the covered distance was the same and no direction needed to be specified. For example "The brown circle loops around the purple rectangle."

4. Wiggling. An object wiggled in place (made small, but visible, up-down/left-right motions without covering distance). Example: "The green trapezoid wiggles."

5. Flashing. An object quickly disappeared and reappeared twice in place, without covering distance. Example: "The green trapezoid flashes."

\section{Table A1}

Visual events in Version 1. Variations of these sentences with pronouns, or meaningful ellipses were accepted as correct utterances.

\begin{tabular}{ccl}
\hline Event set & Trial number & Event \\
\hline 1 & 1 & The green circle moves up one block. \\
1 & 2 & The green circle loops around the purple trapezoid. \\
1 & 3 & The green circle jumps over the purple trapezoid. \\
1 & 4 & The red rectangle loops around the green circle. \\
1 & 5 & The green circle jumps over the red rectangle.
\end{tabular}




\begin{tabular}{|c|c|c|}
\hline Event set & Trial number & Event \\
\hline \multirow[t]{2}{*}{1} & 6 & The purple trapezoid wiggles. \\
\hline & & BREAK \\
\hline 2 & 7 & The purple rectangle jumps over the brown circle \\
\hline 2 & 8 & The purple rectangle moves down three blocks. \\
\hline 2 & 9 & The red diamond moves down three blocks. \\
\hline \multirow[t]{2}{*}{2} & 10 & The purple trapezoid flashes. \\
\hline & & BREAK \\
\hline 3 & 11 & The brown diamond loops around the purple rectangle. \\
\hline 3 & 12 & The purple rectangle loops around the brown diamond. \\
\hline 3 & 13 & The green trapezoid moves up two blocks. \\
\hline 3 & 14 & The brown circle jumps over the green trapezoid. \\
\hline 3 & 15 & The brown circle loops around the green trapezoid. \\
\hline \multirow[t]{2}{*}{3} & 16 & The brown circle jumps under the red rectangle. \\
\hline & & BREAK \\
\hline 4 & 17 & The brown diamond moves right three blocks. \\
\hline 4 & 18 & The red diamond moves down two blocks. \\
\hline 4 & 19 & The red diamond moves left one block. \\
\hline \multirow[t]{2}{*}{4} & 20 & The brown diamond jumps over it [the red diamond]. \\
\hline & & BREAK \\
\hline 5 & 21 & The green trapezoid moves right one block. \\
\hline 5 & 22 & The green trapezoid loops around the red rectangle. \\
\hline 5 & 23 & The red rectangle loops around the green trapezoid. \\
\hline 5 & 24 & The green circle jumps over the red rectangle. \\
\hline \multirow[t]{2}{*}{5} & 25 & The red rectangle jumps under the brown circle. \\
\hline & & BREAK \\
\hline 6 & 26 & The green trapezoid moves down one block. \\
\hline 6 & 27 & The green trapezoid flashes. \\
\hline 6 & 28 & Green trapezoid jumps over the green circle. \\
\hline 6 & 29 & The purple trapezoid loops around the green circle. \\
\hline \multirow[t]{2}{*}{6} & 30 & The purple trapezoid moves down one block. \\
\hline & & BREAK \\
\hline 7 & 31 & The brown diamond moves up one block. \\
\hline 7 & 32 & The purple trapezoid loops around it [The brown diamond]. \\
\hline 7 & 33 & The purple trapezoid jumps over the red diamond. \\
\hline 7 & 34 & The red diamond moves left two blocks. \\
\hline \multirow[t]{2}{*}{7} & 35 & The purple rectangle moves right two blocks. \\
\hline & & BREAK \\
\hline 8 & 36 & The brown circle moves left three blocks. \\
\hline 8 & 37 & The brown circle moves down two blocks. \\
\hline 8 & 38 & The brown circle loops around the purple rectangle. \\
\hline 8 & 39 & The red diamond loops around the purple rectangle. \\
\hline 8 & 40 & The purple rectangle jumps over the brown circle. \\
\hline
\end{tabular}

Brain Stimul. Author manuscript; available in PMC 2015 November 01. 


\begin{tabular}{|c|c|c|}
\hline Event set & Trial number & Event \\
\hline & & BREAK \\
\hline 9 & 41 & The red rectangle moves left one block. \\
\hline 9 & 42 & The purple trapezoid moves up three blocks. \\
\hline 9 & 43 & The purple trapezoid jumps over the green circle. \\
\hline 9 & 44 & The green circle loops around it [the purple trapezoid]. \\
\hline 9 & 45 & The purple trapezoid jumps over the red rectangle. \\
\hline \multirow[t]{2}{*}{9} & 46 & The purple trapezoid moves down one block. \\
\hline & & BREAK \\
\hline 10 & 47 & The red rectangle jumps over the green trapezoid. \\
\hline 10 & 48 & The green trapezoid jumps over the green circle. \\
\hline 10 & 49 & The green trapezoid loops around the green circle. \\
\hline \multirow[t]{2}{*}{10} & 50 & The purple rectangle loops around the green trapezoid. \\
\hline & & BREAK \\
\hline 11 & 51 & The brown diamond jumps over the red rectangle. \\
\hline 11 & 52 & The red rectangle jumps over the brown diamond. \\
\hline 11 & 53 & The red rectangle loops around the brown diamond. \\
\hline 11 & 54 & The brown circle jumps over the purple rectangle. \\
\hline \multirow[t]{2}{*}{11} & 55 & The purple rectangle wiggles. \\
\hline & & BREAK \\
\hline 12 & 56 & The green circle moves down two blocks. \\
\hline 12 & 57 & Green circle moves left two blocks. \\
\hline 12 & 58 & The red diamond jumps over it [the green circle]. \\
\hline 12 & 59 & The red diamond flashes. \\
\hline \multirow[t]{2}{*}{12} & 60 & The red diamond moves right two blocks. \\
\hline & & BREAK \\
\hline 13 & 61 & The purple trapezoid moves left two blocks. \\
\hline 13 & 62 & The brown diamond loops around it [the purple trapezoid]. \\
\hline 13 & 63 & The red diamond jumps over the purple trapezoid. \\
\hline 13 & 64 & The red diamond loops around thr purple trapezoid. \\
\hline \multirow[t]{2}{*}{13} & 65 & The red rectangle moves down three blocks. \\
\hline & & BREAK \\
\hline 14 & 66 & The brown circle moves down two blocks. \\
\hline 14 & 67 & The brown circle moves right three blocks. \\
\hline 14 & 68 & The purple trapezoid moves down one block. \\
\hline 14 & 69 & It [purple trapezoid] jumps over the brown circle. \\
\hline 14 & 70 & The red rectangle loops around the brown circle. \\
\hline \multirow[t]{2}{*}{14} & 71 & The brown circle jumps over the brown diamond. \\
\hline & & BREAK \\
\hline 15 & 72 & The red rectangle moves left two blocks. \\
\hline 15 & 73 & The red rectangle moves up one block. \\
\hline 15 & 74 & The red rectangle jumps over the green trapezoid. \\
\hline 15 & 75 & The red rectangle jumps back over the green trapezoid. \\
\hline
\end{tabular}

Brain Stimul. Author manuscript; available in PMC 2015 November 01. 


\begin{tabular}{|c|c|c|}
\hline Event set & Trial number & Event \\
\hline \multirow[t]{2}{*}{15} & 76 & The green trapezoid wiggles. \\
\hline & & BREAK \\
\hline 16 & 77 & The purple rectangle moves down one block. \\
\hline 16 & 78 & Purple rectangle loops around the green circle. \\
\hline 16 & 79 & The green circle jumps over the purple rectangle. \\
\hline 16 & 80 & The green circle loops around the purple rectangle. \\
\hline \multirow[t]{2}{*}{16} & 81 & The purple rectangle jumps over the red rectangle. \\
\hline & & BREAK \\
\hline 17 & 82 & The brown diamond loops around the brown circle. \\
\hline 17 & 83 & The brown diamond jumps over the brown circle. \\
\hline 17 & 84 & The brown circle wiggles. \\
\hline \multirow[t]{2}{*}{17} & 85 & The red diamond moves left two blocks. \\
\hline & & BREAK \\
\hline 18 & 86 & The brown circle flashes. \\
\hline 18 & 87 & The green trapezoid jumps under the green circle. \\
\hline 18 & 88 & The green circle jumps over the red diamond. \\
\hline 18 & 89 & The green circle loops around the red diamond. \\
\hline \multirow[t]{2}{*}{18} & 90 & The red diamond flashes. \\
\hline & & BREAK \\
\hline 19 & 91 & The green trapezoid moves right two blocks. \\
\hline 19 & 92 & The green trapezoid loops around the red rectangle. \\
\hline 19 & 93 & The red rectangle jumps over it. \\
\hline 19 & 94 & The green trapezoid moves right one block. \\
\hline \multirow[t]{2}{*}{19} & 95 & The red rectangle moves left two blocks. \\
\hline & & BREAK \\
\hline 20 & 96 & The brown diamond moves down three blocks. \\
\hline 20 & 97 & The brown diamond loops around the purple rectangle. \\
\hline 20 & 98 & The brown diamond jumps under the purple rectangle. \\
\hline 20 & 99 & The green trapezoid jumps over the purple rectangle. \\
\hline \multirow[t]{2}{*}{20} & 100 & The purple rectangle jumps over the green trapezoid. \\
\hline & & BREAK \\
\hline 21 & 101 & The purple trapezoid moves left two blocks. \\
\hline 21 & 102 & The brown diamond loops around the purple trapezoid. \\
\hline 21 & 103 & The green trapezoid jumps over the purple trapezoid. \\
\hline 21 & 104 & The brown diamond jumps under the purple trapezoid. \\
\hline \multirow[t]{2}{*}{21} & 105 & The red rectangle wiggles. \\
\hline & & BREAK \\
\hline 22 & 106 & The red rectangle jumps over the red diamond. \\
\hline 22 & 107 & The green circle jumps over the red diamond. \\
\hline 22 & 108 & The red diamond wiggles. \\
\hline 22 & 109 & The purple trapezoid moves left two blocks. \\
\hline 22 & 110 & The brown diamond moves left two blocks. \\
\hline
\end{tabular}

Brain Stimul. Author manuscript; available in PMC 2015 November 01. 


\begin{tabular}{|c|c|c|}
\hline Event set & Trial number & Event \\
\hline & & BREAK \\
\hline 23 & 111 & The purple rectangle flashes. \\
\hline 23 & 112 & The red diamond loops around the green circle. \\
\hline 23 & 113 & The green circle jumps over the purple trapezoid. \\
\hline 23 & 114 & The purple trapezoid jumps over the brown diamond. \\
\hline \multirow[t]{2}{*}{23} & 115 & The brown diamond wiggles. \\
\hline & & BREAK \\
\hline 24 & 116 & The red diamond moves right two blocks. \\
\hline 24 & 117 & The brown circle loops around the red diamond. \\
\hline 24 & 118 & The red diamond jumps over the red rectangle. \\
\hline \multirow[t]{2}{*}{24} & 119 & The red diamond moves down two blocks. \\
\hline & & BREAK \\
\hline 25 & 120 & The brown circle moves down three blocks. \\
\hline 25 & 121 & The brown circle moves left one block. \\
\hline 25 & 122 & The green circle jumps over the red diamond. \\
\hline 25 & 123 & The red diamond loops around the green circle. \\
\hline \multirow[t]{2}{*}{25} & 124 & The green circle flashes. \\
\hline & & BREAK \\
\hline 26 & 125 & The purple rectangle loops around the brown circle. \\
\hline 26 & 126 & The brown circle jumps over the purple rectangle. \\
\hline 26 & 127 & The purple trapezoid moves left three blocks. \\
\hline 26 & 128 & The purple rectangle moves left two blocks. \\
\hline \multirow[t]{2}{*}{26} & 129 & The purple rectangle moves up one block. \\
\hline & & BREAK \\
\hline 27 & 130 & The green circle wiggles. \\
\hline 27 & 131 & The brown diamond loops around the red diamond. \\
\hline 27 & 132 & The purple rectangle loops around the red diamond. \\
\hline 27 & 133 & The purple trapezoid loops around the red diamond. \\
\hline 27 & 134 & The red diamond flashes. \\
\hline
\end{tabular}

\section{Appendix B}

\section{Details of the experimental procedures}

Three phases were completed before the experimental trials. The Speed test was administered to set the optimal speed, and to control for the participants' pre-test characteristics regarding language production. This test was used to exclude participants who differed from the rest in their language abilities (see below). The Orientation and practice phases were completed under stimulation (and sham), for a total of about 5-6 minutes, followed by a 2-minute break. 


\section{Baseline control phase (the Speed test)}

The optimal speed in our paradigm must have two characteristics: (1) Participants should be able to describe the event as it is happening, without having to speak faster than they normally would, and without falling behind and having to reconstruct the event from memory. (2) Participants should fall behind if they wait too long to start speaking, or pause too long in the middle of the sentence. The optimal speed would induce just the right amount of pressure to elicit speech errors, but not too much pressure to disrupt the normal production processes. We first identified the optimal speed with a pilot study, and then pretested all our participants, before they engaged in the main study, to ensure that they could successfully perform the task at that speed. This was necessary in order to preclude possible baseline differences among individuals in the experimental population.

\section{Pilot Study}

The goal of piloting was to find the optimal speed for most participants in the target age group. We started with a slow speed that met criterion 1, but not 2. A script was written which allowed for small increments in speed in Powerpoint. Eight undergraduates from The University of Pennsylvania participated, after completing an unrelated nonverbal experiment. Piloting took less than 10 minutes, during which participants were shown a grid similar to the experimental display, but with only two objects, and were taught how to describe the "moving" action (with direction and number of blocks). The speed of the actions was adjusted by changing the speed parameter in the script, until a parameter was found that met both criteria. We gradually increased the speed, until the participant showed signs of trouble finishing the sentence within the event boundaries. We then dialed back the speed parameter 1 notch and retested the participant. If they were comfortable, that parameter was chosen as their optimal value. This value was very close for all individuals in the pilot population, with participants differing in their ideal parameter by no more than 1 increment. To be conservative, the smaller value (lower speed) was chosen. This parameter was 0.83 , which corresponds to a movement of 1 inch in $1.21 \mathrm{~seconds}(21 \mathrm{~mm} / \mathrm{sec})$, and was set as the optimal speed for the main experiment.

\section{Screening Phase}

To ensure that all participants in the main experiment were comfortable with this speed, and that there were not differences between the two groups in basic language production abilities, everyone completed the "speed test" at $0.83 \mathrm{inch} / \mathrm{sec}$ before they started the experiment. During the 2-minute speed test, participants saw two objects (a black cross and an orange star) move linearly on a grid and described their motions in a manner similar to that of the actual experiment, when the verb was "move"). Participants who were unable to finish their sentences within the boundaries of trials, either due to slow speech rate or too many errors, were excluded from the experiment. One participant failed this test, and was replaced. 


\section{Orientation phase}

First, participants viewed the eight objects in the experiment on a panel, and named each object with its color (e.g., red rectangle). They were corrected if they made any mistakes. Next, each of the five motions was demonstrated separately, using one object (or two in case of "looping around" and "jumping over") on the screen. After each demonstration, participants practiced production of that sentence type using an example. Once the five motions were demonstrated, a review slide reviewed all possible motions, and participants named them one by one. Once it was clear that participants were familiar with all five motions, a full example was demonstrated with all eight objects on the grid, just like the actual experiment. The structure of the example event set was similar to that of an experimental set, with four sentences (trials). Participants viewed the event set and listened to a pre-recorded description of the trials in the set as the events were happening. They then watched the set again, this time without audio, and described the motions themselves. They received feedback and if necessary repeated the example set until they were able to complete that set without errors. Next they moved onto the practice phase.

\section{Practice phase}

In the practice set, participants completed two more event sets, one with four, and one with five sentences. If they made a mistake on their first attempt to describe the event set, they were corrected and the set was repeated. All participants were able to correctly produce the sentences with a maximum of two attempts at each set. The table below shows the average number of times $( \pm \mathrm{SD})$ that subjects in anodal and sham had to repeat the first and the second sets before they were able to produce all trials in each set correctly. The difference between groups was not significant on either set $($ set $1: \mathrm{t}(22)=0.39, \mathrm{p}=0.7$; set 2 : $\mathrm{t}(22)=$ $-0.79, \mathrm{p}=0.44)$.

\begin{tabular}{|c|c|c|}
\hline & Anodal & Sham \\
\hline Trial set 1 & $1.58( \pm 0.51)$ & $1.42( \pm 0.51)$ \\
\hline Trial set 2 & $1.50( \pm 0.52)$ & $1.58( \pm 0.51)$ \\
\hline
\end{tabular}

\section{Why logit mixed models?}

One of the main concerns of tDCS studies is replicability. Even for the most established anodal effects, null effects have been reported (e.g., Liuzzi et al., 2010; Monti et al., 2008). While occasional null effects do not seriously question the past positive effects, it is important to use statistical analyses that properly tease apart stimulation effects from other random effects, introduced into the results by either subjects or items. Appropriate treatment of such random effects not only decreases the chance of reporting false positive results, but allows for generalization over both new participants and new items. Multilevel mixed models (e.g., Jaeger, 2008; Nozari, Kittredge, Dell, \& Schwartz, 2010) are an excellent choice for this purpose. In addition to the benefits mentioned above, when studying an index such as errors, which is inherently categorical (on a given trial, the response was either an 
error or it was correct), as opposed to a continuous measure such as reaction times, methods of categorical data analysis are superior to other methods of analysis. (For a full discussion of the superiority of these models over ANOVA see Agresti, 2013; Jaeger, 2008).

\section{Full results of the analyses with logit mixed models}

\section{Table D1}

The model evaluating the stimulation effect on Fails.

\begin{tabular}{lllll} 
Fixed effects & & & & \\
\hline & coefficient & SE & $\mathbf{z}$ & p-value \\
\cline { 2 - 5 } intercept & -6.04 & 0.46 & -13.05 & $<0.001$ \\
Stimulation & 1.59 & 0.54 & 2.96 & 0.004 \\
Random effects & & & & \\
\hline & & & & \\
Subject intercept & 1.15 & & & \\
Item intercept & 3.99 & & & \\
Stim|item slope & 0.21 & & \\
\hline
\end{tabular}

Table D2

The model evaluating the stimulation effect on the number of errorful sentences

Fixed effects

\begin{tabular}{lllll}
\hline & coefficient & SE & $\mathbf{z}$ & p-value \\
\cline { 2 - 5 } intercept & -2.03 & 0.21 & -9.80 & $<0.001$ \\
Stimulation & 0.72 & 0.28 & 2.55 & 0.01 \\
Random effects & & & & \\
\hline \multicolumn{4}{l}{ Variance } \\
\cline { 2 - 3 } Subject intercept & 0.41 & & & \\
Item intercept & 0.32 & & & \\
Stim|item slope & 0.004 & & & \\
\hline
\end{tabular}

Table D3

The model evaluating the stimulation effect on premature commitments after removing common errors with the perseveration set

Fixed effects

coefficient SE $\quad z \quad$ p-value 


\begin{tabular}{lllll}
\cline { 2 - 4 } intercept & -4.09 & 0.35 & -11.68 & $<0.001$ \\
Stimulation & 1.37 & 0.43 & 3.14 & 0.002 \\
Random effects & & & & \\
\hline & Variance & & \\
\cline { 2 - 3 } & & & \\
Subject intercept & 0.58 & & \\
Stem intercept & 1.58 & & \\
\hline
\end{tabular}

Table D4

The model evaluating the stimulation effect on perseverations after removing common errors with the premature commitment set

Fixed effects

\begin{tabular}{lllll}
\hline & coefficient & SE & $\mathbf{z}$ & p-value \\
\cline { 2 - 5 } intercept & -3.84 & 0.25 & -15.52 & $<0.001$ \\
Stimulation & 0.57 & 0.33 & 1.74 & 0.08 \\
Random effects & & & & \\
\hline & & & & \\
Subject intercept & 0.36 & & \\
Item intercept & 0.42 & & \\
Stimlitem slope & 0.47 & & \\
\hline
\end{tabular}

\section{References}

1. Iyer MB, Mattu U, Grafman J, Lomarev M, Sato S, Wassermann EM. Safety and cognitive effect of frontal DC brain polarization in healthy individuals. Neurology. 2005; 64(5):872-5. [PubMed: 15753425]

2. Nitsche MA, Paulus W. Excitability changes induced in the human motor cortex by weak transcranial direct current stimulation. J Physiol. 2000; 527(3):633-9. [PubMed: 10990547]

3. Nitsche MA, Liebetanz D, Antal A, Lang N, Tergau F, Paulus W. Modulation of cortical excitability by weak direct current stimulation-technical, safety and functional aspects. Suppl Clin Neurophysiol. 2003; 56:255-76. [PubMed: 14677403]

4. Monti A, Cogiamanian F, Marceglia S, Ferrucci R, Mameli F, Mrakic-Sposta S, et al. Improved naming after transcranial direct current stimulation in aphasia. J Neurol Neurosurg Psychiatry. 2008; 79(4):451-3. [PubMed: 18096677]

5. Nozari N, Woodard K, Thompson-Schill SL. Consequences of cathodal stimulation for behavior: when does it help and when does it hurt performance? PloS One. 2014; 9(1):e84338. [PubMed: 24409291]

6. Jacobson L, Koslowsky M, Lavidor M. tDCS polarity effects in motor and cognitive domains: a meta-analytical review. Exp Brain Res. 2012; 216(1):1-10. [PubMed: 21989847] 
7. Fregni F, Boggio PS, Nitsche M, Bermpohl F, Antal A, Feredoes E, et al. Anodal transcranial direct current stimulation of prefrontal cortex enhances working memory. Exp Brain Res. 2005; 166(1): 23-30. [PubMed: 15999258]

8. Baker, J.; Fridriksson, J.; Baker, JM. [cited 2014 Feb 6] Using transcranial direct current stimulation (tDCS) to treat persons with aphasia. 2010. Available from: http://aphasiology.pitt.edu/archive/ 00002119/01/viewpaper.pdf

9. Fertonani A, Rosini S, Cotelli M, Rossini PM, Miniussi C. Naming facilitation induced by transcranial direct current stimulation. Behav Brain Res. 2010; 208(2):311-8. [PubMed: 19883697]

10. Flöel A, Rösser N, Michka O, Knecht S, Breitenstein C. Noninvasive brain stimulation improves language learning. J Cogn Neurosci. 2008; 20(8):1415-22. [PubMed: 18303984]

11. Fridriksson J, Richardson JD, Baker JM, Rorden C. Transcranial Direct Current Stimulation Improves Naming Reaction Time in Fluent Aphasia A Double-Blind, Sham-Controlled Study. Stroke. 2011; 42(3):819-21. [PubMed: 21233468]

12. Marangolo P, Fiori V, Di Paola M, Cipollari S, Razzano C, Oliveri M, et al. Differential involvement of the left frontal and temporal regions in verb naming: a tDCS treatment study. Restor Neurol Neurosci. 2013; 31(1):63-72. [PubMed: 23142815]

13. Flöel A. Non-invasive brain stimulation and language processing in the healthy brain. Aphasiology. 2012; 26(9):1082-102.

14. Hamilton RH, Chrysikou EG, Coslett B. Mechanisms of aphasia recovery after stroke and the role of noninvasive brain stimulation. Brain Lang. 2011; 118(1):40-50. [PubMed: 21459427]

15. Holland R, Crinion J. Can tDCS enhance treatment of aphasia after stroke? Aphasiology. 2012; 26(9):1169-91. [PubMed: 23060684]

16. Schlaug G, Marchina S, Wan CY. The use of non-invasive brain stimulation techniques to facilitate recovery from post-stroke aphasia. Neuropsychol Rev. 2011; 21(3):288-301. [PubMed: 21842404]

17. Sparing R, Dafotakis M, Meister IG, Thirugnanasambandam N, Fink GR. Enhancing language performance with non-invasive brain stimulation - a transcranial direct current stimulation study in healthy humans. Neuropsychologia. 2008; 46(1):261-8. [PubMed: 17804023]

18. Ross LA, McCoy D, Wolk DA, Coslett H, Olson IR. Improved proper name recall by electrical stimulation of the anterior temporal lobes. Neuropsychologia. 2010; 48(12):3671-4. [PubMed: 20659489]

19. Fiori V, Cipollari S, Di Paola M, Razzano C, Caltagirone C, Marangolo P. tDCS stimulation segregates words in the brain: evidence from aphasia. Front Hum Neurosci [Internet]. 2013; 7 Available from: http://www.ncbi.nlm.nih.gov/pmc/articles/PMC3682157/.

20. Manenti R, Petesi M, Brambilla M, Rosini S, Miozzo A, Padovani A, et al. Efficacy of semanticphonological treatment combined with tDCS for verb retrieval in a patient with aphasia. Neurocase. 2014:1-11. ahead-of-print.

21. Wang J, Wu D, Chen Y, Yuan Y, Zhang M. Effects of transcranial direct current stimulation on language improvement and cortical activation in nonfluent variant primary progressive aphasia. Neurosci Lett. 2013; 549:29-33. [PubMed: 23800543]

22. Liuzzi G, Freundlieb N, Ridder V, Hoppe J, Heise K, Zimerman M, et al. The involvement of the left motor cortex in learning of a novel action word lexicon. Curr Biol. 2010; 20(19):1745-51. [PubMed: 20888226]

23. De Vries MH, Barth AC, Maiworm S, Knecht S, Zwitserlood P, Flöel A. Electrical stimulation of Broca's area enhances implicit learning of an artificial grammar. J Cogn Neurosci. 2010; 22(11): 2427-36. [PubMed: 19925194]

24. Fiori V, Cipollari S, Caltagirone C, Marangolo P. "If two witches would watch two watches, which witch would watch which watch?" tDCS over the left frontal region modulates tongue twister repetition in healthy subjects. Neuroscience. 2014; 256:195-200. [PubMed: 24184977]

25. Nozari N, Thompson-Schill SL. More attention when speaking: Does it help or does it hurt? Neuropsychologia. 2013; 51(13):2770-80. [PubMed: 24012690]

26. Marangolo P, Fiori V, Calpagnano MA, Campana S, Razzano C, Caltagirone C, et al. tDCS over the left inferior frontal cortex improves speech production in aphasia. Front Hum Neurosci [Internet]. 2013; 7 Available from: http://www.ncbi.nlm.nih.gov/pmc/articles/PMC3764371/. 
27. Nozari N, Dell GS. Feature migration in time: Reflection of selective attention on speech errors. J Exp Psychol Learn Mem Cogn. 2012; 38(4):1084. [PubMed: 22268910]

28. Haller S, Radue EW, Erb M, Grodd W, Kircher T. Overt sentence production in event-related fMRI. Neuropsychologia. 2005; 43(5):807-14. [PubMed: 15721193]

29. Etard O, Mellet E, Papathanassiou D, Benali K, Houdé O, Mazoyer B, et al. Picture naming without Broca's and Wernicke's area. Neuroreport. 2000; 11(3):617-22. [PubMed: 10718324]

30. Nozari N, Schwartz M. Fluency of Speech Depends on Executive Abilities: Evidence for Two Levels of Conflict in Speech Production. Procedia-Soc Behav Sci. 2012; 61:183-4.

31. Antal A, Nitsche MA, Paulus W. Transcranial direct current stimulation and the visual cortex. Brain Res Bull. 2006; 68(6):459-63. [PubMed: 16459203]

32. Jaeger TF. Categorical data analysis: Away from ANOVAs (transformation or not) and towards logit mixed models. J Mem Lang. 2008; 59(4):434-46. [PubMed: 19884961]

33. Nozari N, Kittredge AK, Dell GS, Schwartz MF. Naming and repetition in aphasia: Steps, routes, and frequency effects. J Mem Lang. 2010; 63(4):541-59. [PubMed: 21076661]

34. Barr DJ, Levy R, Scheepers C, Tily HJ. Random effects structure for confirmatory hypothesis testing: Keep it maximal. J Mem Lang. 2013; 68(3):255-78.

35. Sandson J, Albert ML. Perseveration in behavioral neurology. Neurology. 1987; 37(11):17361736. [PubMed: 3670611]

36. Fischer-Baum S, Rapp B. Underlying cause (s) of letter perseveration errors. Neuropsychologia. 2012; 50(2):305-18. [PubMed: 22178232]

37. Dell GS, Schwartz MF, Martin N, Saffran EM, Gagnon DA. Lexical access in aphasic and nonaphasic speakers. Psychol Rev. 1997; 104(4):801. [PubMed: 9337631]

38. Nozari N, Dell GS, Schwartz MF. Is comprehension necessary for error detection? A conflictbased account of monitoring in speech production. Cognit Psychol. 2011; 63(1):1-33. [PubMed: 21652015]

39. Müller SV, Johannes S, Wieringa B, Weber A, Müller-Vahl K, Matzke M, et al. Disturbed monitoring and response inhibition in patients with Gilles de la Tourette syndrome and co-morbid obsessive compulsive disorder. Behav Neurol. 2003; 14(1-2):29-37. [PubMed: 12719636] 
We propose the first systematic paradigm for studying effects of tDCS on sentence production.

Anodal tDCS of PFC reliably decreased the rate of omitted and errorful sentences. A reliable decrease was found for errors due to premature commitment. A marginal effect was found for perseveratory errors. 


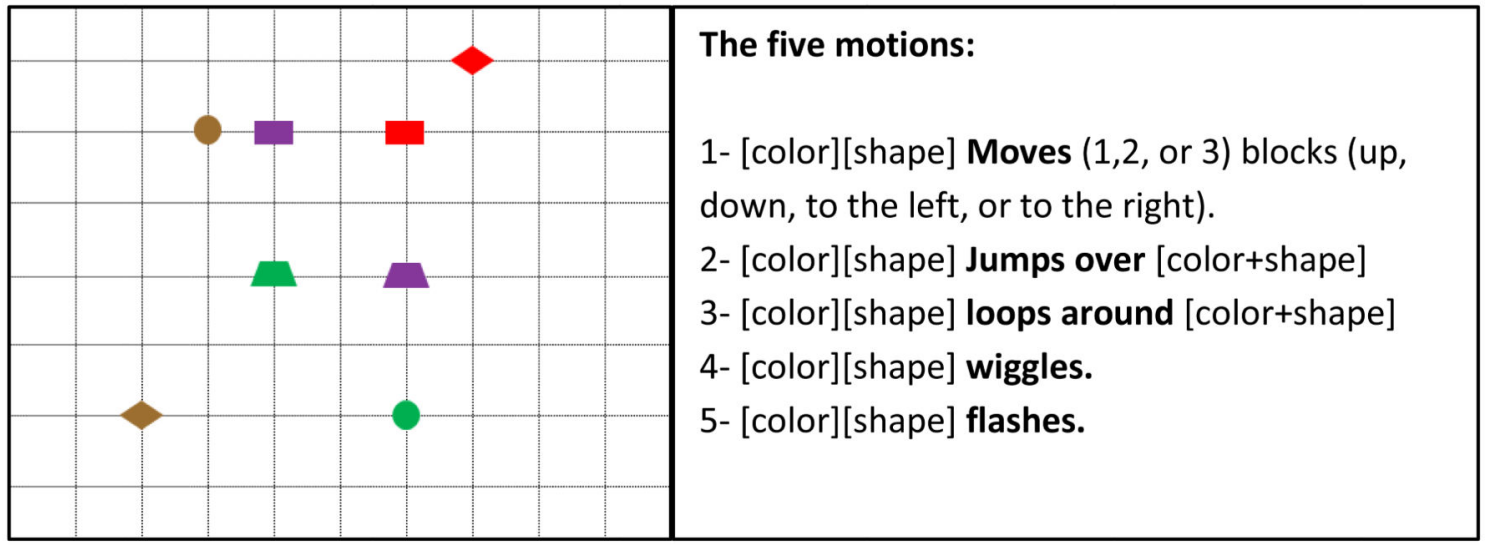

Figure 1.

A screenshot of the experiment as participants viewed it (version 1), along with the five possible motions. 


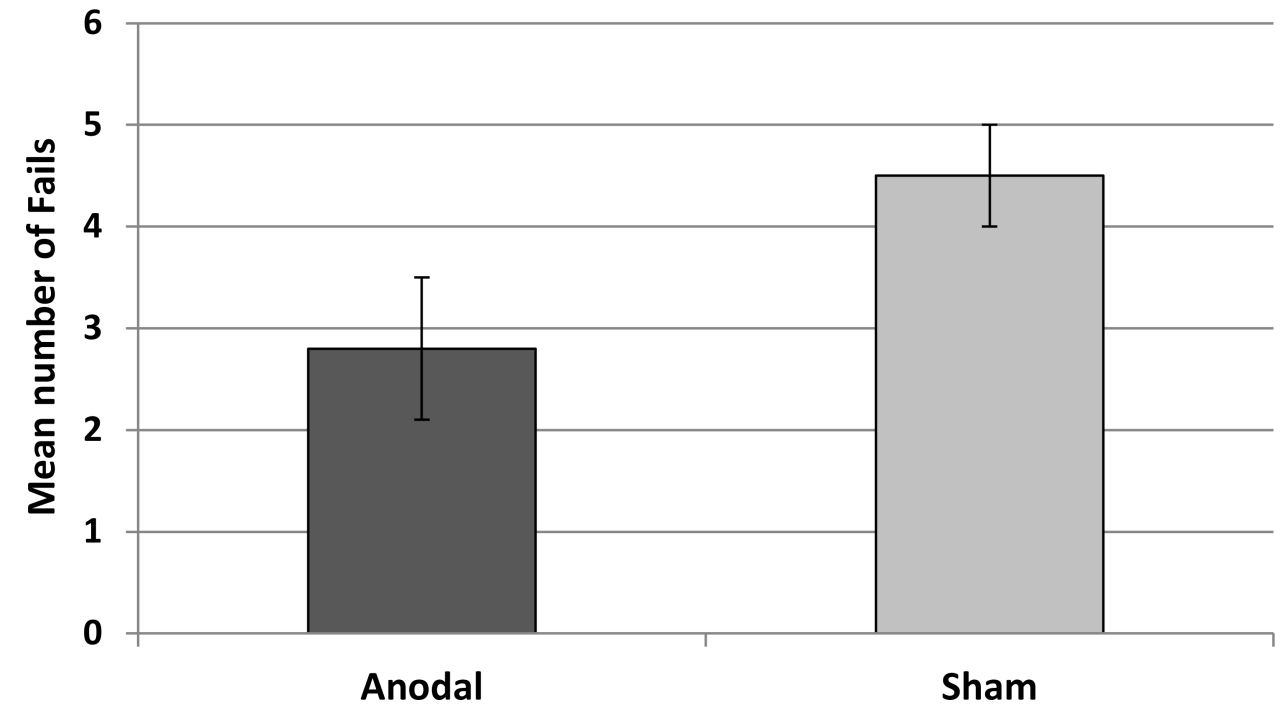

Figure 2.

Average number of Fails $( \pm \mathrm{SE})$ in Anodal vs. sham. See text for definition. 

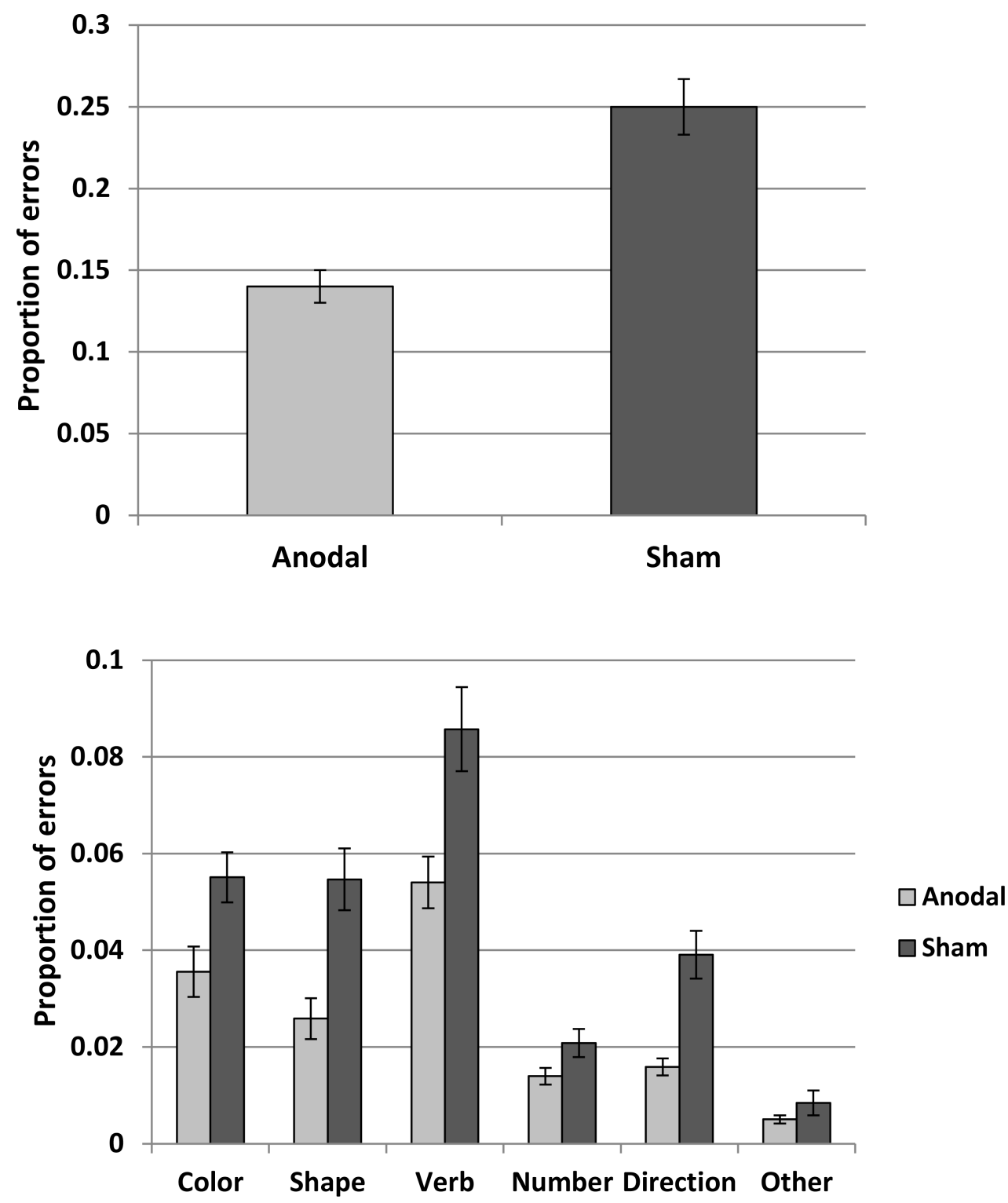

Figure 3.

Mean proportion of sentences with at least one error $( \pm \mathrm{SE})$ in the anodal and sham conditions. The upper panel shows the average of all error types. The lower panel breaks down errors by type. This figure shows that across the board there are fewer errors in the anodal vs. sham condition. 
(a)

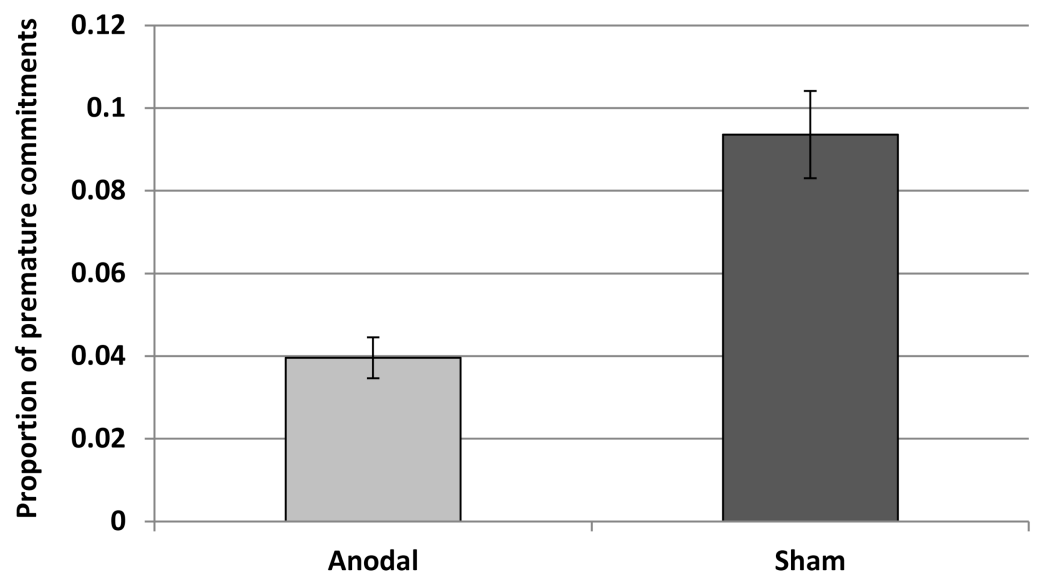

(b)

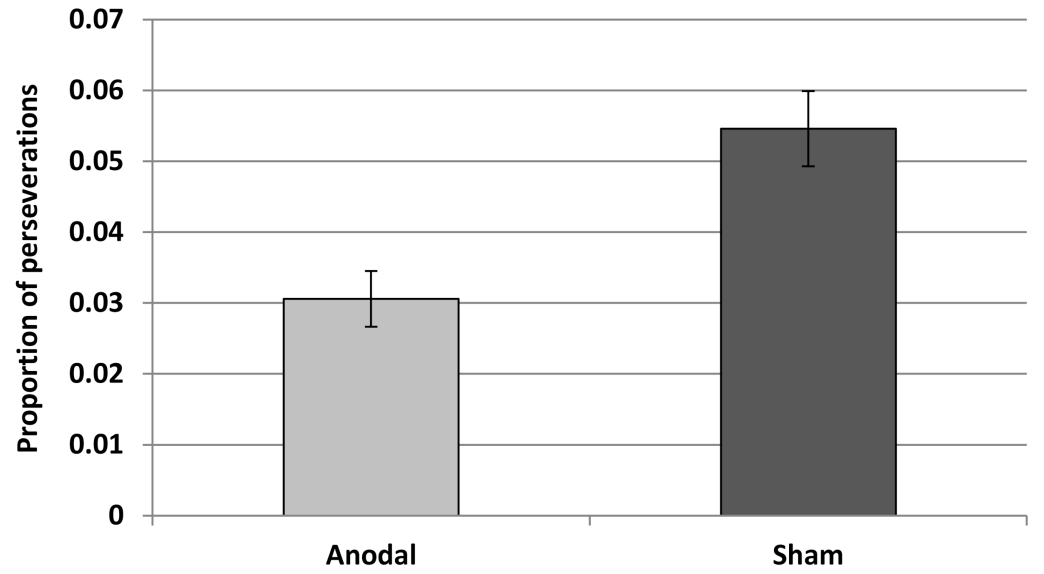

Figure 4.

Mean proportion $( \pm \mathrm{SE})$ of the two error types: (a) premature commitments, after the removal of perseveratory errors, in anodal and sham conditions. (b) Perseveratory errors, after the removal of premature commitments, in anodal and sham conditions. Potentially, all uttered sentences provide an opportunity for a perseveratory error. See text for definition. 


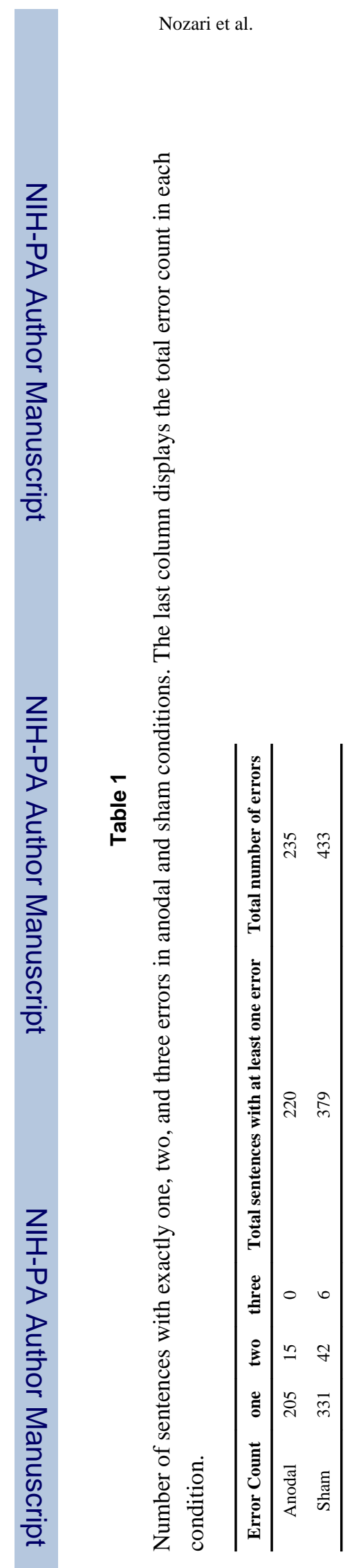

Brain Stimul. Author manuscript; available in PMC 2015 November 01. 


\section{Table 2}

Number of errors for perseverations and premature commitments under anodal and sham stimulation, after removing the ambiguous trials.

\begin{tabular}{lc}
\hline Stimulation & Number of errors \\
\hline Perseveration & \\
Anodal & 47 \\
Sham & 76 \\
\hline Premature commitments & \\
Anodal & 33 \\
Sham & 66 \\
\hline
\end{tabular}

